\title{
Artemisinin Loaded mPEG-PCL Nanoparticle Based Photosensitive Gelatin Methacrylate Hydrogels for the Treatment of Gentamicin Induced Hearing Loss
}

This article was published in the following Dove Press journal:

International Journal of Nanomedicine

\author{
Xiaohua $\mathrm{Li}^{1}$ \\ Yanchun Wang ${ }^{2}$ \\ Feilong $X u^{\prime}$ \\ Feng Zhang' \\ Ying $\mathrm{Xu}^{3}$ \\ Lei Tang ${ }^{3}$ \\ Thomas J Webster (1D ${ }^{4}$ \\ 'Department of Otolaryngology, The \\ First Affiliated Hospital of Zhengzhou \\ University, Zhengzhou 450052, People's \\ Republic of China; ${ }^{2}$ Department of \\ Chinese Medicine, Henan Provincial \\ People's Hospital, People's Hospital of \\ Zhengzhou University, People's Hospital \\ of Henan University, Zhengzhou, Henan \\ 450003, People's Republic of China; \\ ${ }^{3}$ College of Pharmacy, Jiangsu University, \\ Zhenjiang 2120I3, People's Republic of \\ China; ${ }^{4}$ Department of Chemical \\ Engineering, Northeastern University, \\ Boston, MA 02115, USA
}

Objective: Artemisinin (ART) is a natural anti-malarial sesquiterpene lactone which has the ability to treat and activate the CLRN1 pathway to play a pivotal role in hearing loss and hair cell function. To investigate the therapeutic effect of ART in hearing loss induced by gentamicin (GM), an ART-loaded poly(ethylene glycol)-b-poly(e-caprolactone) mPEG-PCL nanoparticle-based photosensitive hydrogel was developed and tested in this study.

Materials and Methods: Artemisinin-loaded mPEG-PCL nanoparticles (mPEG-PCL-ARTNPs) were prepared by a double emulsion method and the formulation was optimized by an orthogonal experimental design. The particle size, zeta potential, morphology and in vitro dissolution of the mPEG-PCL-ART-NPs were well characterized. Biocompatibility of the mPEG-PCL-ART-NPs were tested on HeLa cells with an MTT assay. The photo-crosslinkable biodegradable gelatin methacrylate (GelMA) hydrogel was prepared and its physicochemical properties (such as substitution, photocrosslinking efficiency, cell viability morphology, mechanical and swelling properties) were evaluated. Finally, mPEG-PCL-ART-FITC-NPs, loaded mPEG-PCL-ART-NPs, and loaded mPEG-PCL-ART-NPs-GelMA hydrogels were fabricated and a GM toxicity-induced guinea pig ear damage model was established to determine the effectiveness of the materials on returning auditory function and cochlea pathomorphology.

Results: The zeta potential of the mPEG-PCL-ART-NPs was about $-38.64 \pm 0.21 \mathrm{mV}$ and the average size was $167.51 \pm 1.87 \mathrm{~nm}$ with an encapsulation efficacy of $81.7 \pm 1.46 \%$. In vitro release studies showed that the $\mathrm{mPEG}$-PCL-ART-NPs possessed a sustained-release effect and the MTT experiments showed good biocompatibility properties of the drug-loaded nanoparticles. The results indicated that the 5\% GelMA with MA-4\% hydrogel had a better crosslinking density and 3D structure for drug loading and drug delivery than controls. Skin penetration results showed that the mPEG-PCL-ART-NPs increased adhesive capacity and avoided fast diffusion in the skin. Most importantly, auditory brainstem response results indicated that the mPEG-PCL-ART-NPs-GelMA hydrogel alleviated hearing loss induced by GM.

Conclusion: These results suggested that the presently fabricated mPEG-PCL-ARTNPs-GelMA hydrogels are promising formulations for the treatment of hearing loss induced by GM and lay the foundation for further clinical research of inner ear induction therapy.

Keywords: artemisinin, mPEG-PCL nanoparticles, GelMA hydrogel, hearing loss, gentamicin

\section{Introduction}

Hearing impairment is a serious disease $\mathrm{e}^{1-3}$ wherein human health and the quality of life are seriously affected by ear problems. ${ }^{4}$ In order to treat hearing impairment,

Correspondence: Xiaohua Li;

Thomas J Webster

Tel +860I3837I60836;

Tel + I 6173736585

Email kfxiaohua@I26.com;

th.webster@neu.edu 
there is a need to seek an effective way to prevent ear infections. $^{5}$ Any injuries to the cochlea could lead to irreversible hearing impairment. ${ }^{6}$ Cochlea injury caused by ototoxic drugs is regarded as a major issue for all types of hearing impairment and has to be addressed now. ${ }^{7}$

In particular, gentamicin, a kind of antibiotic which can kill bacterial, especially gram-negative ones, ${ }^{8}$ has been considered a popular choice for treating infections due to its low cost and high efficacy. ${ }^{9}$ However, gentamicin can also cause hearing loss mainly due to its ototoxic and nephrotoxic effects. ${ }^{10,11}$ Previous studies have shown that gentamicin has the capacity to catalyze free radical generation via a chemical reaction that involves the formation of an iron complex, which is crucial for normal mitochondrial function. ${ }^{12,13}$ Free-radical formation has been accepted as a potential cause of ototoxicity, while antioxidants usually weaken amino glycoside-induced hearing loss. ${ }^{14}$

Artemisinin (ART) and its derivatives are powerful biomedical agents known for their capability to rapidly decrease levels of plasmodium parasites in the blood of patients with malaria. ${ }^{15}$ As a sesquiterpene lactone endoperoxide, ART is a natural compound that exists in the plant Artemisia annua. ${ }^{16}$ Also, ART has received considerable attention mainly because of its strong antimalarial effect. ${ }^{17}$ In recent times, several studies have been conducted on ART, namely to elucidate its antiprotozoal, ${ }^{18}$ antibacterial, ${ }^{9}$ antiviral ${ }^{19}$ and anticancer ${ }^{20}$ activities. In spite of its high efficacy for treating various diseases, the literature suggests that the pharmacological and pharmacokinetic characteristics of ART are affected by its low solubility in aqueous solution, poor bioavailability and short half-life. ${ }^{21}$

In order to improve its pharmacokinetic properties, ART has been loaded into various drug delivery carriers. ${ }^{22}$ Among these studies, the incorporation of ART into nanoparticle carriers has been deemed the best approach to yield an improved biological outcome of a drug. ${ }^{22,23}$ Nanoencapsulation of drugs is a widely developed technique which has been applied to improve drug controlled release delivery systems. ${ }^{24}$ Previously, ART has been incorporated into various nanoparticle systems (eg, lipids, polymers, inorganic, cyclodextrin-based, etc.) showing improved drug stability as well as prolonged sustained release strengthening the bioavailability of a drug. ${ }^{25}$ The encapsulation of hydrophobic drugs into nanoparticles has the potential to improve a drug-loaded nanoparticle system for many applications, such as for intravenous delivery. ${ }^{26}$
Therefore, amphiphilic nanoparticles have a great potential to deliver hydrophobic drugs and may be useful for ART.

Specifically, polycaprolactone-polyethylene glycolpolycaprolactone or PCL-PEG-PCL nanoparticles, a triblock PCL-PEG-PCL copolymer, has been selfassembled into nanoparticles via the fabrication of a coreshell structure: a hydrophobic PCL core and a hydrophilic PEG shell. ${ }^{27}$ Further, loading of hydrophobic drugs into PCL-PEG-PCL copolymers could improve drug distribution and drug targeting for sustained-release over a predetermined time in vitro and in vivo. ${ }^{28}$ Moreover, the biocompatibility, recyclability and ease of preparation of PCL-PEG-PCL copolymers has enhanced its application for a wide range of drug delivery systems. ${ }^{29}$

Polymeric hydrogels have also been reported to exhibit great potential as drug carriers ${ }^{30}$ as they are hydrated threedimensional (3D) networks of natural or synthetic polymers that can be formulated to match the physical and chemical properties of all human tissues, ${ }^{31}$ which are derived from both collagen $^{32}$ and denatured collagen (or gelatin). ${ }^{33,34}$ These kinds of native extracellular matrix proteins from natural polymers are especially advantageous as carriers of drugs due to their excellent biocompatibility and biodegradability. ${ }^{32,35}$

Gelatin methacrylate (GelMA) is derived from gelatin and consists of a degraded natural extracellular matrix. ${ }^{36}$ It has been crosslinked covalently and in an irreversible manner in the presence of a photoinitiator and exposure to ultraviolet (UV) light. ${ }^{37,38}$ GelMA hydrogels have many excellent properties such as low cost, ease of production, natural cell binding ability and adjustable degradation. ${ }^{39,40}$ In order to improve the water solubility of ART, here, we encapsulated ART into mPEG-PCL-NPs and a GelMA hydrogel. The physicochemical properties of the ART-loaded mPEG-PCL nanoparticle (mPEG-PCL-ART-NP)-based GelMA hydrogel were characterized, while their in vitro drug release, cytocompatibility, skin penetration and histological evaluation were also investigated. We hypothesize here that the encapsulation of ART into mPEG-PCL to form nanoparticles could serve as a controlled release system for targeted drug delivery, and the embedded nanoparticles in the GelMA hydrogel could be locally released to the injury providing a potential treatment for gentamicin-induced hearing loss.

\section{Materials and Methods \\ Materials}

Artemisinin (ART) was obtained from Merck, Darmstadt, Germany (Art No. 820,354). Poly( $\varepsilon$-caprolactone)-b-poly 
(ethylene glycol) (PCL-PEG6000), gelatin, poly(vinyl alcohol), the photoinitiator (PI) and methacrylic anhydride (MA) were all purchased from Sigma-Aldrich (USA). Phosphate-buffer saline (PBS) and other solutions were prepared with deionized water (18.2 $\mathrm{M} \Omega \mathrm{cm}$, Millipore, USA). HeLa cells (JK-CS0290), fibroblasts (JK-R3203), and hair cells (JK-R1225) were purchased from JingKang Bio Co., LTD (Shanghai, China). All the experimental protocols were approved by the Jiangsu University Animal Ethics and Experimentation Committee and followed the principles of the laboratory and animal care committee of the University.

\section{Preparation of Artemisinin-Loaded mPEG-PCL Nanoparticles}

Preparation of Artemisinin Nanoparticles (mPEG-PCLART-FITC-NP) and Empty Nanoparticles (mPEG-PCLNP) by a Double Emulsion Method

ART-loaded mPEG-PCL nanoparticles were prepared via a double emulsion method. ${ }^{28}$ Briefly, PEG-PCL (40 mg) and ART $(10 \mathrm{mg})$ were dissolved in $2 \mathrm{~mL}$ of DCM as the oil phase. Then, $200 \mathrm{~mL}$ of water or a FITC (5 g/L) solution was added as the aqueous phase. The aqueous and oil phases (empty mPEG-PCL or mPEG-PCL with ART) were mixed evenly, while the colostrum was prepared by ultrasonic treatment with a cell grinder (JY99-IID, Ningbo Biotechnology Co., Ltd., Ningbo, China) for $3 \mathrm{~min}$. The obtained colostrum was added to $10 \mathrm{~mL}$ of a $1 \%$ PVA water solution, while double emulsion was formed under ultrasound for $5 \mathrm{~min}$ with a power of $10 \%$. Next, the double emulsion was dispersed in $10 \mathrm{~mL}$ of a $0.3 \%$ PVA solution and then dried via a rotary evaporator. Afterwards, the nanoparticles were collected using a centrifuge at low temperature and high speed $(10,000 \mathrm{rpm})$ for $10 \mathrm{~min}$. Finally, the nanoparticle solutions were formed by dissolving the formulation with $1 \mathrm{~mL}$ of deionized water and the mPEG-PCL-ART-FITC-NP and the empty nanoparticles mPEG-PCL-NP were obtained by freeze-drying under a pressure of $14 \mathrm{~Pa}$ at $-80{ }^{\circ} \mathrm{C}$ to separate all of the remaining solvents.

\section{The Orthogonal Experimental Design}

According to our preliminary research, four parameters viz., the concentration of the emulsifier (A), PEG-PCL content (B), the volume ratio of the water phase to the oil phase (C) and the emulsifying time (D) had the most significant effect on the preparation of the mPEG-PCLART-FITC-NPs. In order to obtain the optimal synthesis conditions, the experiments were orthogonally designed by arranging the aforementioned four factors with three levels for each as shown in Table 1. Nine different sets of experiments were conducted under different parameter combinations according to the standard $L_{9}\left(3^{4}\right)$ table as indicated in Table 2. Each experiment was repeated twice.

Further orthogonal experiments were carried out to optimize the prescription. The factors of the orthogonal experiment were: concentration of emulsifier (A), dosage of $\mathrm{mPEG}-\mathrm{PCL}(\mathrm{B})$, volume ratio of external water phase to oil phase (C), and emulsification time (D).

\section{Particle Size Analysis}

The particle size distribution of the optimized ART-loaded PEG-PCL nanoparticles was determined via a dynamic light scattering (DLS) technique using a nano/Zeta sizer (Malvern Instruments, Worcestershire, UK, model Nano ZS). The nanosystem of ART-loaded PEG-PCL nanoparticles were observed by transmission electron microscopy (TEM, JEM-2100F; JEOL, Tokyo, Japan) after negative staining of the preparations with uranyl acetate or by cryoTEM after quench-freezing a thin film of particle suspension in liquid ethane.

\section{Determination of Encapsulation Efficiency (EE\%)}

Encapsulation efficiency (EE \%) was calculated per the following equation:

Encapsulation efficiency $(\mathrm{EE} \%)=($ weight of drug in $\mathrm{mPEG}-\mathrm{PCL}-\mathrm{NP}) /($ weight of initial drug $) \times 100 \%$

\section{Drug Release Study}

A drug release study was carried out to estimate the release profile of ART from the mPEG-PCL nanosystem under different $\mathrm{pH}$ values. Briefly, $20 \mathrm{mg}$ of freeze-dried nanoparticles or ART were dispersed in $3 \mathrm{~mL}$ of deionized water and then the whole suspension was placed into a dialysis sac (12 $\mathrm{kDa}$ ) and incubated at $37^{\circ} \mathrm{C}$ while being immersed in $35 \mathrm{~mL}$ of phosphate buffer saline (PBS, $\mathrm{pH} \mathrm{1.2,} 7.0$ and 7.8). Then, at programmed time intervals, $1 \mathrm{~mL}$ of the dialysate was

Table I Orthogonal Test Factor Levels

\begin{tabular}{|l|l|l|l|}
\hline Factor & $\begin{array}{l}\text { Level } \\
\text { I }\end{array}$ & $\begin{array}{l}\text { Level } \\
\mathbf{2}\end{array}$ & $\begin{array}{l}\text { Level } \\
\mathbf{3}\end{array}$ \\
\hline $\begin{array}{l}\text { The concentration of the emulsifier (\%) } \\
\text { mPEG-PCL contents }(\mathrm{mg})\end{array}$ & 0.5 & 1 & 1.5 \\
$\begin{array}{l}\text { The volume ratio of the water phase to } \\
\text { the oil phase (W/O) }\end{array}$ & $3: 1$ & $5: 1$ & $7: 1$ \\
Emulsifying time (min) & 4 & 8 & 12 \\
\hline
\end{tabular}


Table 2 Experimental Design and Results of the Orthogonal Design $L_{9}\left(3^{4}\right)$

\begin{tabular}{|l|l|l|l|l|l|l|l|}
\hline No. & A & B & C & D & Particle Size nm & Entrapment Rate EE\% & Comprehensive Scores \\
\hline I & I & I & I & I & 198.34 & 50.4 & 45.93 \\
2 & I & 2 & 2 & 2 & 190.71 & 66.8 & 57.21 \\
3 & I & 3 & 3 & 3 & 265.29 & 69.3 & 45.70 \\
4 & 2 & I & 2 & 3 & 167.03 & 77.5 & 67.84 \\
5 & 2 & 2 & 3 & 1 & 286.17 & 79.1 & 59.00 \\
6 & 2 & 3 & 1 & 2 & 230.49 & 82.4 & 47.93 \\
7 & 3 & 1 & 3 & 2 & 257.36 & 70.7 & 58.35 \\
8 & 3 & 2 & 1 & 3 & 206.43 & 73.2 & 63.32 \\
9 & 3 & 3 & 2 & 1 & 196.74 & 78.6 & \\
\hline K1 & 49.61 & 53.90 & 52.42 & 52.42 & & & \\
K2 & 58.52 & 54.52 & 54.96 & 54.96 & & & \\
K3 & 56.53 & 56.25 & 47.21 & 57.30 & & & \\
R & 8.91 & 2.35 & 7.75 & 4.88 & & & \\
\hline
\end{tabular}

Note: Comprehensive scores $=0.5 \times 100 \times(1$-particle size/maximum particle size $)+0.5 \times 100 \times($ Entrapment rate/maximum entrapment rate) .

taken out and replaced with fresh PBS $(1 \mathrm{~mL})$. The concentration of ART in the dialysate was determined by a UV-Vis spectrophotometer at $290 \mathrm{~nm}$. All of the release studies were carried out in triplicate.

\section{Cellular Uptake Assay}

Fibroblasts were seeded in 48-well plates at a seeding density of $2 \times 10^{4}$ cells/well for $24 \mathrm{~h}$ to allow for cell attachment. The supernatants in each well were replaced with fresh culture medium containing mPEG-PCL-ART-FITC-NPs at final concentrations of $150 \mu \mathrm{g} / \mathrm{mL}$ and continuously cultured for $10 \mathrm{~min}$. In another experimental group, the time-dependent uptake was also studied. The cells were incubated with mPEGPCL-ART-FITC-NPs (final concentration of $150 \mu \mathrm{g} / \mathrm{mL}$ ) for 2, 4, 6, 8, 12 and $24 \mathrm{~h}$. Then, the RPMI 1640 medium containing 10\% FBS was replaced while culturing for an additional $2 \mathrm{~h}$. After incubation, all of the samples were washed three times with PBS and observed under fluorescence microscopy (TE-2000U, Nikon, Tokyo, Japan) while the number of FITC positive cells was calculated by using ImageJ (National Institutes of Health, USA).

\section{Cell Viability}

Cytotoxic activities of blank mPEG-PCL-NPs and mPEGPCL-ART-FITC-NPs towards HeLa and hair cells were evaluated via MTT assays. Briefly, the cells were seeded in 96-well plates with $100 \mu \mathrm{L}$ of medium at a density of $1 \times 10^{5}$ cells per well. After $24 \mathrm{~h}$ of incubation, the cells were washed with PBS. Then, $100 \mu \mathrm{L}$ of fresh medium containing various concentrations of samples $(1,5$ and $10 \mu \mathrm{g} / \mathrm{mL})$ was added $(\mathrm{n}=5$ per group). After incubation for $48 \mathrm{~h}, 20 \mu \mathrm{L}$ of the MTT solution
$(0.5 \mathrm{mg} / \mathrm{mL})$ was added into each well and incubated for another $4 \mathrm{~h}$ to quantify the live cells. Then, the culture medium was removed. Aliquots $(150 \mu \mathrm{L})$ of DMSO were added into each well. Finally, the 96-well plates were placed in a microplate reader to measure absorbance at $490 \mathrm{~nm}$ :

$$
R G R(\%)=\frac{\text { Abs } 490 \text { test }}{\text { Abs } 490 \text { control }}
$$

All of the results were estimated based on six individual experiments and expressed as the mean $\pm \mathrm{SD}$.

\section{Gelatin Methacryloyl Synthesis}

Gelatin methacryloyl (GelMA) was synthesized by following a previous protocol. ${ }^{37}$ Briefly, $10 \mathrm{~g}$ of gelatin type A was dissolved at $10 \mathrm{w} / \mathrm{v} \%$ in PBS at $60{ }^{\circ} \mathrm{C}$ under vigorous stirring. Methacrylic anhydride was then slowly added under constant stirring at $50{ }^{\circ} \mathrm{C}$ until a concentration of $1,2,4$ and 8 vol \% was reached and allowed to react for $2 \mathrm{~h}$. Following a $2 \mathrm{X}$ dilution with warm PBS $\left(40^{\circ} \mathrm{C}\right)$ to stop the reaction, the solution was immediately dialyzed against deionized water using a $12-14 \mathrm{kDa}$ cutoff dialysis membrane at $40{ }^{\circ} \mathrm{C}$ for 1 week to remove salts, side products and unreacted chemicals. After lyophilizing the dialyzed solution, a white porous foam of GelMA was obtained and stored at $-80{ }^{\circ} \mathrm{C}$ for further use.

\section{Characterization of GelMA Hydrogels GelMA Substitution Test}

Standard determination: GelMA (1\%, 2\%, 4\% and 8\%) with different degrees of substitution was gathered to prepare $1 \mathrm{~g} / \mathrm{L}$ sample solutions, and $1 \mathrm{~mL}$ of each solution was 
placed in a $15 \mathrm{~mL}$ centrifuge tube. In addition, a blank control was established with deionized water. Next, $1 \mathrm{~mL}$ of a $4 \% \mathrm{NaHCO}_{3}$ solution was added to each centrifuge tube prior to full dissolution at $50{ }^{\circ} \mathrm{C}$. Then, $0.1 \%$ of a trinitrobenzene sulfonic acid (TNBS) solution was prepared and $1 \mathrm{~mL}$ was added to each centrifuge tube for the reaction to occur. After a few seconds, different concentrations of orange appeared in the sample tube, while the blank tube was light yellow. All of the centrifugal tubes were placed in a constant temperature gas bath oscillator, while a light avoidance reaction was performed at $40{ }^{\circ} \mathrm{C}$ for $2 \mathrm{~h}$. Afterwards, $1 \mathrm{~mol} / \mathrm{L}$ of a hydrochloric acid solution $(5 \mathrm{~mL})$ was added into each centrifuge tube to cause hydrolysis. Consequently, the blank tube was almost colorless, while the sample tube was light yellow. The centrifuge tube was placed at $99^{\circ} \mathrm{C}$ and protected from light for $2 \mathrm{~h}$. Finally, all of the sample solutions were added to the 96-well plate, with $200 \mu \mathrm{L}$ per well set up in triplicate. The 96-well plate was placed on an automatic microplate and the absorbances of different samples were detected at a wavelength of $340 \mathrm{~nm}$.

Gelatin standard curve: A gelatin solution $(0.2,0.4$, 0.6, 0.8 and $1 \mathrm{~g} / \mathrm{l}$ ) was prepared as the standard solution, while $1 \mathrm{~mL}$ of the solution was placed in a $15 \mathrm{~mL}$ centrifuge tube. Next, the samples were repeated to obtain the absorbance of a gelatin standard solution. The standard curve was drawn with the concentration of the gelatin solution as the abscissa $(0 \mathrm{~g} / \mathrm{L}$ gelatin solution as deionized water) and the absorbance as the ordinate.

\section{Photocrosslinking Experiment}

GelMA solutions at 3, 5 and $10 \mathrm{w} / \mathrm{v} \%$ in phosphate buffered saline (PBS) containing a PI at $0.5 \mathrm{w} / \mathrm{v} \%$ were prepared. Photocrosslinking was carried out by exposing the GelMA solutions under UV light (OmniCure S2000, Excelitas Technologies) at a power of $1 \mathrm{~W} / \mathrm{cm}^{2}$ and a distance of $8 \mathrm{~cm}$ between the UV output and the UV exposure time was 60 s. Next, GelMA with different degrees of substitution were obtained, which was useful for the subsequent experiments.

\section{Cell Viability}

Cell viability of the GelMA hydrogel at different concentrations and degrees of substitution were analyzed by staining them with the Live/Dead kit (Lift Technologies) at 24,72 and $120 \mathrm{~h}$ in accordance with the manufacturer's instructions. The samples were stained using phalloidin/ 4',6-diamidino-2-phenylindole (DAPI) for F-actin/nuclei, followed by observation under an inverted fluorescence microscope (Nikon Ti-E, Japan). Consequently, the green cells were live cells while the red cells were dead cells. The number of live cells ( $\mathrm{Nl}$ ) and the number of dead cells (Nd) in the cell fluorescence images were calculated by ImageJ to determine the cell survival rate (Rs) as follows:

$$
R_{s}=\frac{N_{l}}{N_{l}+N_{d}} \times 100 \%
$$

\section{The Morphology of the GelMA Hydrogel}

GelMA hydrogel scaffolds were obtained using a cryostat and freeze-dried. A scanning electron microscope (SEM, FEI, Quanta 200, Oregon, USA) was used to characterize the morphology of the GelMA hydrogel.

\section{Mechanical Property Test}

In the experiment, a dynamic mechanical analyzer (ElectroForce, TA Instruments, USA) was used to test the mechanical properties of GelMA hydrogels with different degrees of substitution and concentrations by compression and tensile tests.

In the tensile experiment, three hydrogel samples with different degrees of substitution (GelMA concentration of $5 \%$ ) were placed in a $4 \times 10 \times 4 \mathrm{~cm}$ mold (block), photocrosslinked under $365 \mathrm{~nm}$ ultraviolet light $\left(1 \mathrm{~W} / \mathrm{cm}^{2}\right.$, OmnicureS2000, 60s), and then stretched at a speed of $1 \mathrm{~mm} / \mathrm{min}$. The formula for determining its strain, $\varepsilon$, and stress, $\sigma$, were as follows:

$$
\begin{gathered}
\varepsilon=\frac{l-l_{0}}{l_{0}}=\frac{\Delta l}{l_{0}} \\
\sigma=\frac{F}{A_{0}}
\end{gathered}
$$

where $l_{0}$ is the initial length of the specimen, 1 is the length after tensile deformation, $\Delta \mathrm{l}$ is the tensile deformation variable of the material, $\mathrm{F}$ and $\mathrm{A}_{0}$ are the load on the specimen and the initial cross-sectional area of the tensile specimen, respectively.

In the compression test, the GelMA hydrogel was lightly cured into a cylindrical shape (thickness of $2 \mathrm{~mm}$ and diameter of $12 \mathrm{~mm}$ ) in a mold. Each cylinder sample was placed between two compression plates, while the experiment was performed at a displacement rate of $1 \mathrm{~mm} / \mathrm{min}$ with the compression stress-strain formula being the same as the tensile stress-strain formula. The Young's Modulus and compression modulus of the hydrogels were calculated 
according to the stress-strain curve of the linear strain range of the $0-10 \%$ slope.

\section{Swelling Property Test}

The immersed GelMA hydrogel with different degrees of substitution in PBS buffer was placed in a $37{ }^{\circ} \mathrm{C}$ incubator and was allowed to fully swell. Next, the GelMA hydrogel was taken out before filter paper was applied to absorb the water on the surface after $24 \mathrm{~h}$. Afterwards, the mass of the swelling hydrogel $\left(\mathrm{W}_{1}\right)$ was accurately weighed and placed in an ultra-low temperature refrigerator prior to freeze-drying until a new weight $\left(\mathrm{W}_{2}\right)$ was attained. The swelling degree (swelling ratio, SR) of the GelMA hydrogel was calculated according to the following formula:

$$
\mathrm{SR}=\frac{W_{1}-W_{2}}{W_{2}}
$$

All samples were measured 3 times in parallel.

\section{Preparation of mPEG-PCL-ART-FITC-NPs Loaded GelMA Hydrogel and Transdermal Tests}

GelMA solutions at $5 \mathrm{w} / \mathrm{v} \%$ in PBS were prepared prior to the addition of ART $(1 \mathrm{mg} / \mathrm{mL})$ to the GelMA solution. Subsequently, the mPEG-PCL-ART-FITC-NPs loaded GelMA hydrogels (mPEG-PCL-ART-FITC-NPs-GelMA hydrogel) were obtained.

The TK-20A transdermal diffusion tester was used to evaluate the transdermal effect of the preparations using vertical Franz diffusion cells (Shanghai Kaikai Technology Trade Co., Ltd., China) with a diffusional area of $1.77 \mathrm{~cm}^{2}$ and a receptor chamber volume of $7 \mathrm{~mL}$. The temperature and stirring rate of the diffusion apparatus were set at $32{ }^{\circ} \mathrm{C}$ and $300 \mathrm{r} / \mathrm{min}$, respectively. Samples of pure ART $(1 \mathrm{mg} / \mathrm{mL})$, the mPEG-PCL-ART-FITC-NPs solution $(1 \mathrm{mg} / \mathrm{mL})$ and the mPEG-PCL-ART-FITC-NPs-GelMA hydrogel $(1 \mathrm{mg} / \mathrm{mL})$ were prepared. The guinea pig skin was sandwiched between the diffusion cells with the stratum corneum side up and the dermal side in contact with the receiver compartment. A $2 \mathrm{~mL}$ corresponding drug solution was added into the donor compartment, while the control was treated with the vehicle only. At predetermined time intervals, an aliquot $(1 \mathrm{~mL})$ of the sample was withdrawn from each receptor chamber and replaced with the equivalent volume of the fresh receiver solution. After the receiving liquid was filtered through a $0.45 \mu \mathrm{m}$ microporous filter membrane, the content was determined by High Performance Liquid Chromatography (HPLC).
The HPLC conditions were: the column: Hypersil BDSC18 $(4.6 \mathrm{~mm} \times 150 \mathrm{~mm}, 5 \mu \mathrm{m})$; mobile phase: methanol: water $(70: 30, \mathrm{~V} / \mathrm{V})$; flow rate: $1 \mathrm{~mL} \cdot \mathrm{min}^{-1}$; detection wavelength: $210 \mathrm{~nm}$; column temperature: $25^{\circ} \mathrm{C}$ and injection volume: $20 \mu \mathrm{L}$.

\section{Measurement of the Auditory Brainstem Responses (ABR)}

The measurements of the threshold shift of ABR were performed based on previously published protocols [16]. A mixture of ketamine $(40 \mathrm{mg} / \mathrm{kg})$ and xylazine $(10 \mathrm{mg} / \mathrm{kg})$ was used to anesthetize guinea pigs which were kept warm on heating pads throughout the procedure. The reference, ground and active electrodes were placed beneath the postmeasured auricle skin, the skin of sacrococcygeal region and the calvaria skin, respectively. The responses to the presentations of 1024 click were filtered, amplified and synchronously averaged. Each stimulus was set at an initial pressure level (SPL) of $100 \mathrm{~dB}$, and the intensity systematically decreased by a $5-\mathrm{dB}$ interval until a discernible ABR disappeared. The threshold of ABR was determined as a series of stimulus (30-3000) that produced a detectable response.

The guinea pigs were randomly divided into four groups and 5 guinea pigs were selected per group, viz., group A (control group), group B (GM Group), group C (GM+ mPEG-PCL-ART-NPs group) and group D (GM+ mPEGPCL-ART-NPs-GelMA hydrogel group).

Group A: An equal amount of normal saline (normal saline was $3 \mathrm{~mL} \cdot \mathrm{kg}^{-1} \cdot \mathrm{d}^{-1}$ ), intramuscular injection on the inner thigh and the guinea pigs were weighed daily, while the dosage was calculated by weight, with the treatment taking place for 14 consecutive days.

Group B: A gentamicin sulfate (GM) injection, $120 \mathrm{mg} \cdot \mathrm{kg}^{-1} \cdot \mathrm{d}^{-1}$, was given to the animals prior to daily weighing with the other procedure performed as stated above.

Group C: A gentamicin sulfate (GM) injection, $120 \mathrm{mg} \cdot \mathrm{kg}^{-1} \cdot \mathrm{d}^{-1}$, was determined based on the body weight. The intramuscular injection of the thigh lasted for 14 days. From the first day of injection, mPEG-PCLART-NPs $10 \mathrm{mg} / \mathrm{kg}$ was given orally to the pigs by gavage.

Group D: A gentamicin sulfate (GM) injection (120 $\mathrm{mg} \cdot \mathrm{kg}^{-1} \cdot \mathrm{d}^{-1}$ ) was given to the animals, who underwent similar procedures as described above except that after the first day of injection, the guinea pigs were administered with $0.5 \mathrm{~mL}$ of the $4 \mathrm{mg} / \mathrm{mL}$ mPEG-PCL-ART-NPs loaded 
GelMA hydrogel via infusion into the ear while UV irradiation was performed.

\section{Histological Examinations}

For histological examination, guinea pigs were sacrificed at 14 day, while the guinea pig cochlea were harvested. Guinea pig cochlea were fixed in $3.7 \%$ neutral buffered formalin, processed routinely into paraffin, sectioned into $4 \mu \mathrm{m}$ slices, and stained with hematoxylin and eosin (H\&E). The histology was performed in a blinded fashion by professional personnel in the medical college of Jiangsu University. The samples were examined with a microscope (Olympus BX61 Inverted Microscope) under bright-field.

\section{Results and Discussion Determination of the Optimal Preparation Parameters}

The analysis for the optimization of the preparation parameters for the mPEG-PCL-NPs was previously highlighted in Tables 1 and 2. Based on the analysis, it could be seen that the mPEG-PCL-NP particle size was remarkably affected by the concentration of the emulsifier. Meanwhile, the mPEG-PCL-NP particle size was also markedly influenced by the volume ratio of the water phase to the oil phase $(\mathrm{W} / \mathrm{O})$, but its significance ( $\mathrm{R}$ value) was lower than the concentration of the emulsifier (Table 2). Using parameter A (the concentration of the emulsifier), the particle size and $\mathrm{EE} \%$ of $\mathrm{A} 2$ were the highest with EE\% being more than that of $\mathrm{A} 1$ and $\mathrm{A} 3$. Thus, A2 was chosen as one of the optimal preparation conditions. In terms of parameter B (mPEG-PCL content), the particle size and $\mathrm{EE} \%$ of $\mathrm{B} 3$ were the highest. Similarly, both $\mathrm{C} 2$ and D3 were selected as the best preparation conditions. Therefore, $\mathrm{A}_{2} \mathrm{~B}_{3} \mathrm{C}_{2} \mathrm{D}_{3}$ (namely the concentration of the emulsifier is $1 \%$, PEG-PCL content is $60 \mathrm{mg}$, the volume ratio of the water phase to the oil phase is $5: 1$, and the emulsifying time is $12 \mathrm{~min}$ ) was chosen as the optimal condition for the preparation of the nanoparticles. In confirmatory experiments using these optimal conditions, the particle size of the samples was $167.51 \pm 1.87$ $\mathrm{nm}$, while the PDI and zeta potential of the optimized ART-loaded mPEG-PCL-NPs were $0.207 \pm 0.017$ and $-38.64 \pm 0.21 \mathrm{mV}$, respectively (Figure 1). Also, the EE $\%$ for the ART-loaded mPEG-PCL nanoparticles reached $81.7 \pm 1.46 \%$.

According to the particle size analysis, the average size of the mPEG-PCL-ART-NPs was $167.51 \pm 1.87 \mathrm{~nm}$, PDI was $0.207 \pm 0.017$, and the potential was $-38.64 \pm 0.21 \mathrm{mV}$. In this experiment, nanoparticles were prepared using the double emulsion method. The physical and chemical parameters of the nanoparticles were examined. TEM images showed the morphological characteristics of the nanoparticles which were nearly spherical with a diameter of $210 \mathrm{~nm}$. After the incorporation of ART into the nanoparticles, the particle size obviously increased.

\section{In vitro Release}

In order to investigate the effect of the chemical and biochemical features on the release of ART from the mPEG-PCL nanoparticles, a release study was performed on the drug-loaded mPEG-PCL nanoparticles in a neutral water medium $(\mathrm{pH}=7.0)$, PBS solution $(\mathrm{pH}=7.8)$ and hydrochloric acid $(\mathrm{HCl})$ solution $(\mathrm{pH}=1.2)$. As shown in Figure 2, pure ART was rapidly released into the water, PBS and $\mathrm{HCl}$ solution, suggesting that there was a burst release in the initial period which negatively affected its sustainable-release into the media (Figure 2A). Likewise,

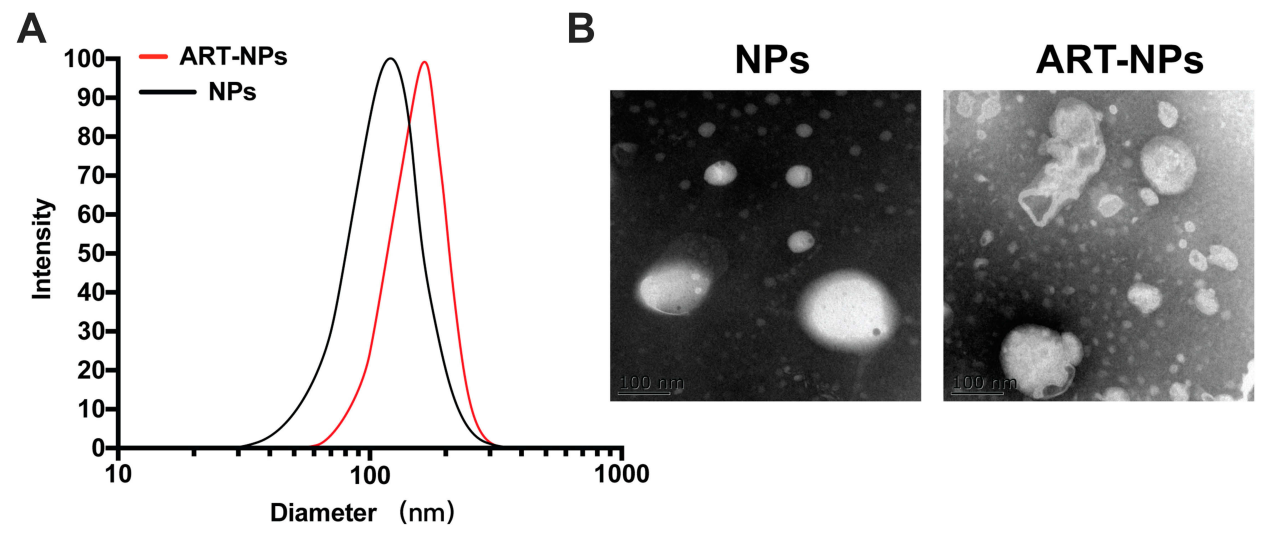

Figure I The particle size and TEM photographs of the mPEG-PCL-ART-NPs. (A) The diameter of nanoparticles via DLS and (B) TEM photographs of the nanoparticles. 
A

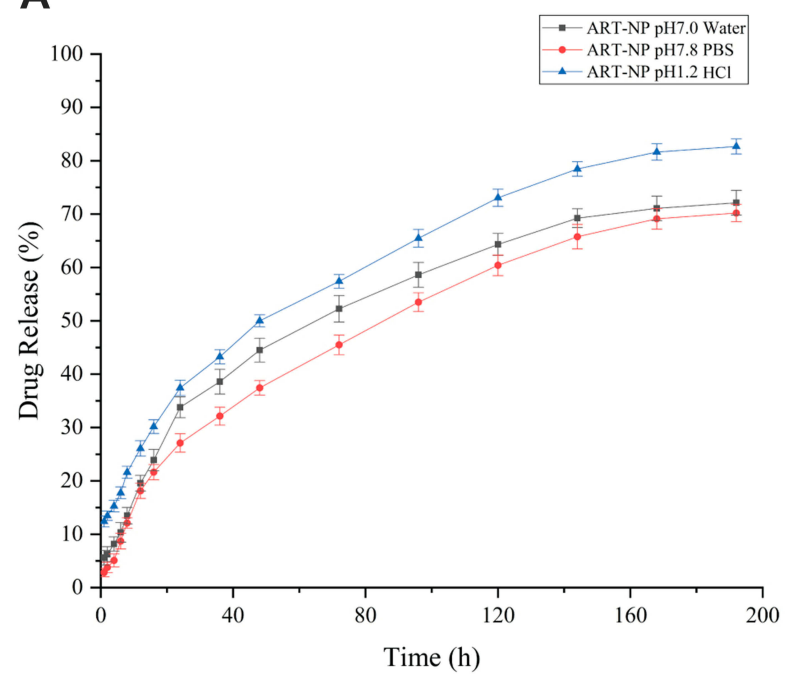

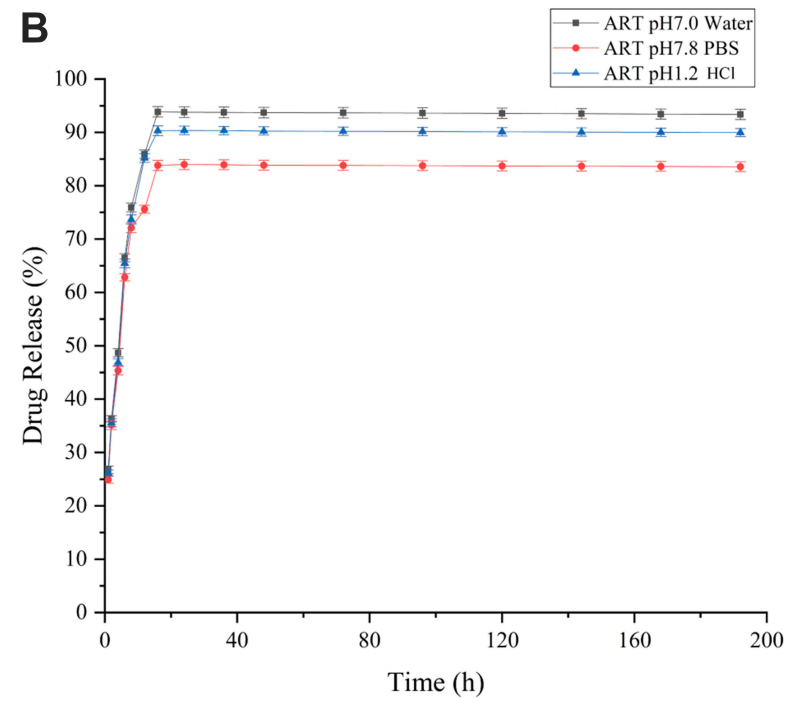

B

- ART-NP-GELMA pH7.0 Water - ART-NP-GELMA pH7.8 PBS
$\triangle$ ART-NP-GELMA pH1.2 $\mathrm{HCl}$

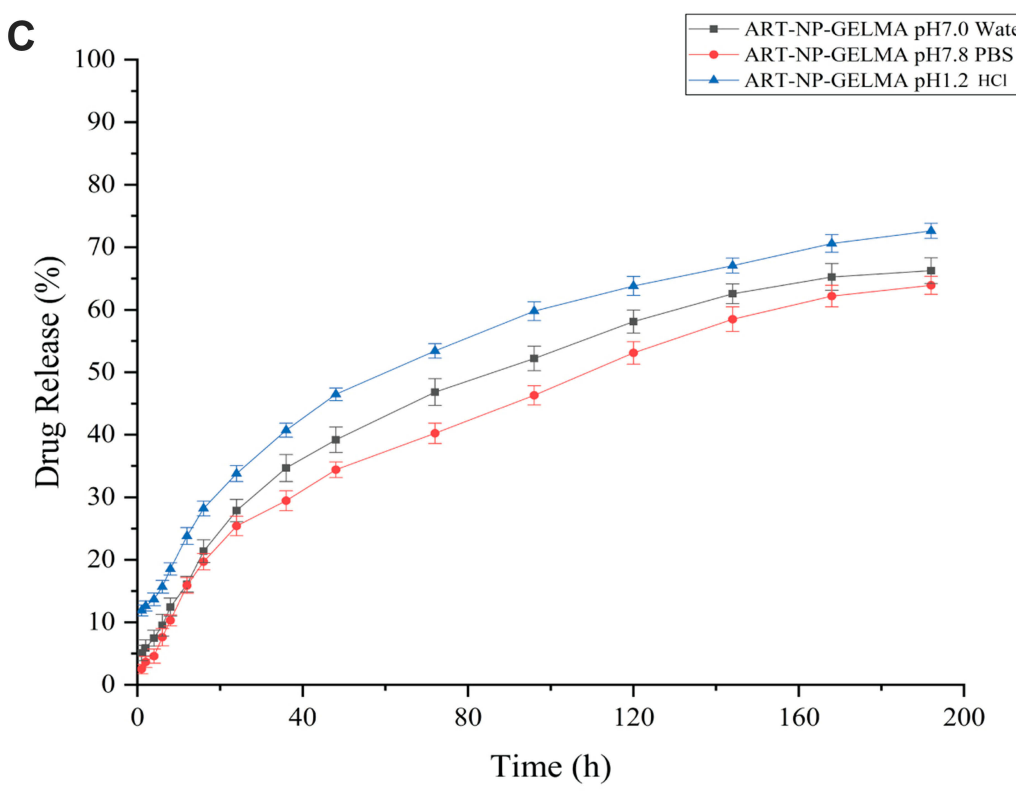

Figure 2 The release profiles of (A) ART, (B) mPEG-PCL-ART-NPs, and (C) ART-NPs-GelMA in different release media.

the mPEG-PCL-ART-NPs showed a similar release pattern in different media (Figure 2B). However, with time, the drug release rates in the different media continuously increased. At $200 \mathrm{~h}$, the release amount reached $85 \%$ in the $\mathrm{HCl}$ solution with no observable burst release during the initial period. This occurrence lies in the $\mathrm{pH}$ sensitivity of the release rate of ART from the mPEG-PCL because the copolymer is degradable under acidic conditions by hydrolysis $^{25}$ and the ART did not achieve full release which was mainly attributed to the drug small molecule agglomeration. Therefore, these results demonstrated that the mPEG-PCL-NPs could be used as a promising drug carrier for the sustained-release of medications in vitro.

\section{Cellular Uptake Assay}

To investigate cellular uptake and intracellular distribution of mPEG-PCL-ART-FITC-NPs, FITC was employed as a model drug for intracellular tracing. The released FITC was observed with confocal laser scanning microscopy (Zeiss LSM880 with Airyscan, Germany) after incubation with fibroblasts. After incubation with ART-FITC and mPEG-PCL-ART-FITC-NPs, the cells displayed a wellspread morphology, indicating good cytocompatibility. As depicted in Figure 3, the green fluorescence from the internalized FITC illustrated in the confocal image clearly indicates that ART was released from both ART-FITC and 


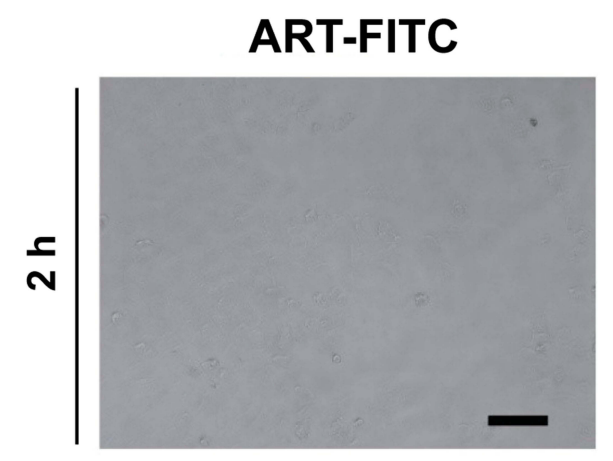

\section{MPEG-PCL-ART-FITC}
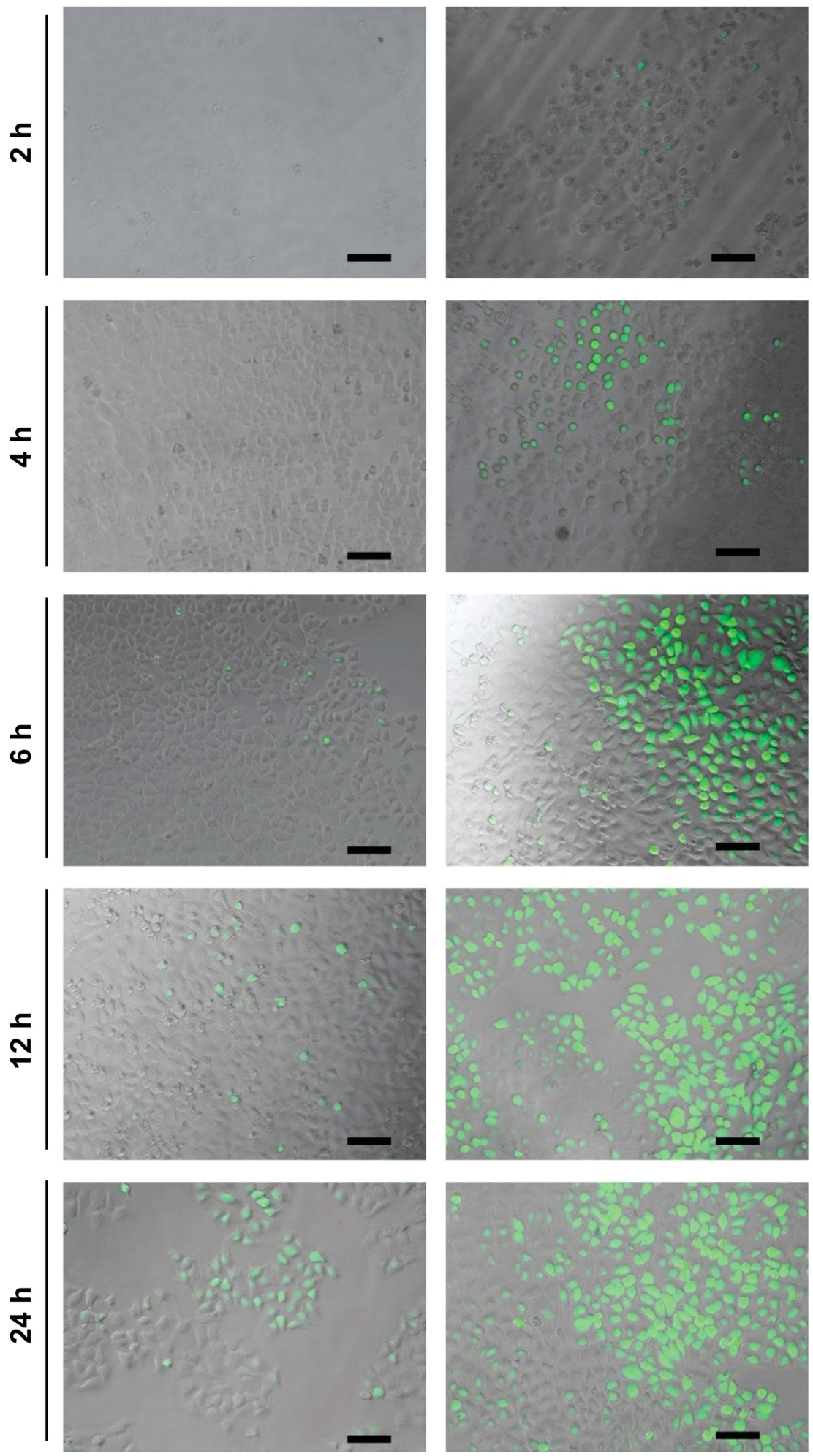

Figure 3 Representative confocal microscopy images of ART-FITC and mPEG-PCL-ART-FITC-NPs endocytosed by fibroblast cells for 2, 4, 6, 12 and $24 \mathrm{~h}$. The scale bar is $50 \mu \mathrm{m}$. 
mPEG-PCL-ART-FITC-NPs carriers into the fibroblasts. It could be observed that after $6 \mathrm{~h}$ of incubation, only a small amount of fluorescent particles was found in the cytoplasm of fibroblasts. Meanwhile, as time progressed, the number of fluorescent particles in the cytoplasm of fibroblasts increased significantly after $12 \mathrm{~h}$ and $24 \mathrm{~h}$ of incubation. These results illustrate that ART-FITC and mPEG-PCLART-FITC-NPs could be effectively internalized into live cells while endocytosis efficiency increased along with incubation time. However, mPEG-PCL-ART-FITC-NPs displayed markedly lower cell uptake efficiency than that of the ART-FITC at $6 \mathrm{~h}, 12 \mathrm{~h}$, and $24 \mathrm{~h}$ incubation period, respectively. Collectively, these findings suggest that ART may be endocytosed with mPEG-PCL possessing a strong potential as a drug carrier for fibroblasts.

\section{Biocompatibility of the Nanoparticles}

Biological safety is very important for the application of nanoparticles as the next generation of biomaterials. In this regard, an MTT assay was performed to investigate the cellular biocompatibility of ART and the optimized mPEGPCL-ART-NPs. Considering the fact that both PEG and PCL do not exhibit high cytotoxicity when used as drug carriers, mPEG-PCL-ART-NPs showed a negligible effect on fibroblasts (Figure 4). However, cell viability decreased after incubation with high concentrations of ART and the optimal mPEG-PCL-ART-NPs. Probably, although requiring further investigation, nanoparticles entered into the cells and ART was released at high concentrations leading to decreased cell viability. ${ }^{25}$ These studies specify that high concentrations of ART and mPEG-PCL-ART-NPs may have a very remarkable anticancer effect, specifically for HeLa cells. Notwithstanding, the viability of hair cells

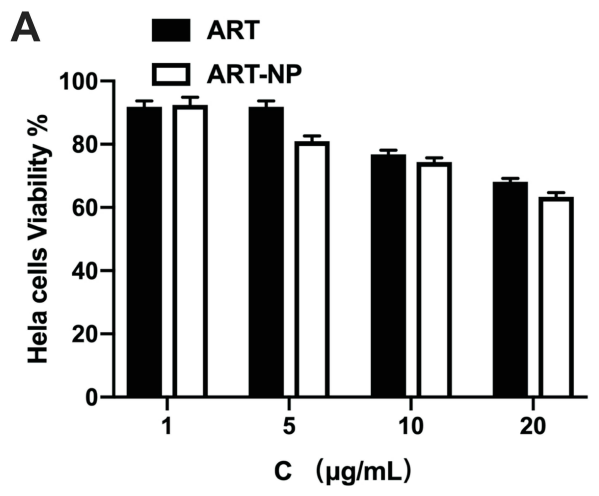

increased when the ART concentration increased (1-10 $\mu \mathrm{g} / \mathrm{mL}$ ), while the increase was significant for the $\mathrm{mPEG}-$ PCL-ART-NPs group compared to ART (Figure 4B). When the concentration of ART increased to $20 \mu \mathrm{g} / \mathrm{mL}$, the hair cells were inhibited. Therefore, these results imply that low concentrations of ART and optimal concentrations of mPEG-PCL-ART-NPs had excellent biocompatibility and did not cause any levels of toxicity to hair cells.

\section{The Degree of Substitution and Gelation Ability of the GelMA Hydrogel}

The degree of substitution has a significant influence on the physicochemical properties of the GelMA hydrogel. Figure 5 shows the gelation ability mapping of the various GelMA compositions (3\%: 1, 4, 7, and10: 5\%: 2, 5, 8, and $11 ; 7 \%: 3,6,9$ and 12) and MA concentrations (1-3: 1\% MA, 4-6: 2\% MA, 7-9: 4\% MA, 10-12: 8\% MA). Thus, such results suggest that the degree of substitution of GelMA could increase to over $60 \%$. The gelation ability of the different degrees of substitution of GelMA compositions and MA concentrations are shown in Figure 5B. At high MA concentrations, it can be observed that the GelMA solution was converted to a hydrogel, which could attach to the wall of the bottle when the glass bottle was turned upside down. However, the GelMA could not form gelatin at low concentrations of both GelMA and MA. Figure 5A showed that GelMA with 26.2\%, 41.5\%, $53.2 \%$ and $67.6 \%$ degrees of substitution, respectively, created customized GelMA polymers with different levels of MA. When considering the selection of GelMA concentration based on the above results, the best available technology, economic viability, and cost effectiveness, we selected 5\% and 10\% GelMA to perform the subsequent studies.

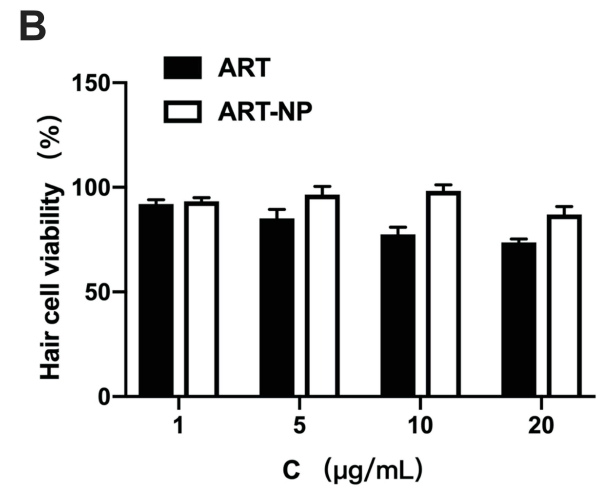

Figure 4 In vitro viability of cells in the presence of ART and mPEG-PCL-ART-NPs for I, 5, 10 and $20 \mu g / \mathrm{mL}$, respectively. (A) Hela cells and (B) Hair cells. 
A

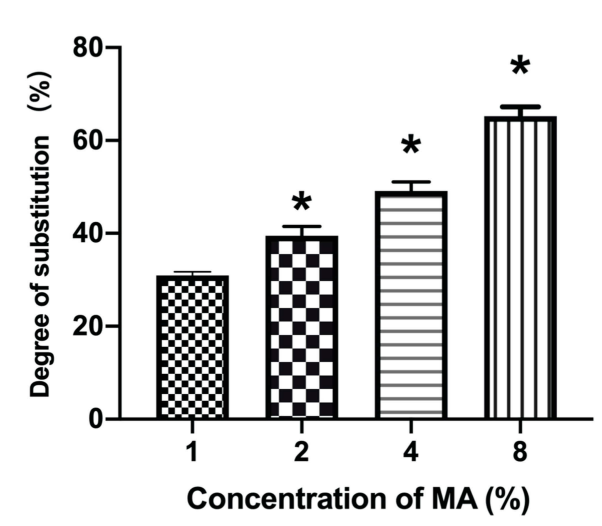

\section{B Photocrosslinking \\ 12. Not photocrosslinking}

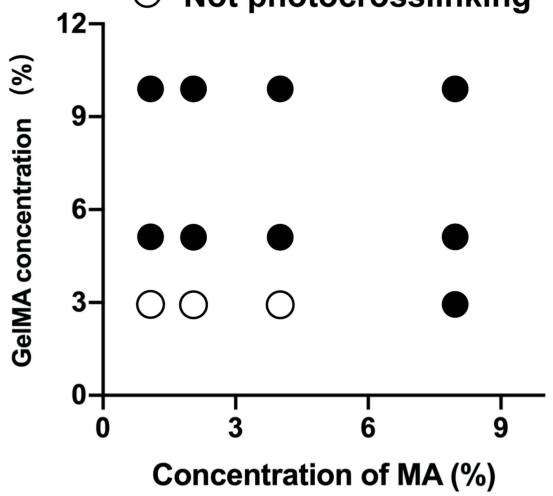

C

Figure 5 The degree of substitution and gelation ability of the GelMA hydrogels. The degree of substitution of the GelMA with various MA levels (A). Gelation ability mapping of the various GeIMA compositions (3\%: I, 4, 7, and 10; 5\%: 2, 5, 8, and II; 7\%: 3, 6, 9 and I2) and MA concentrations (I-3: I\% MA, 4-6: 2\% MA, 7-9: 4\% MA, 10-12: 8\% MA) (B). Photographs showing the gelation ability of different degrees of substitution of the GelMA solution, before and after UV light crosslinking (C). ${ }^{*}<0.01$ compared to the lowest concentration.

\section{Optimization of MA Concentration}

The cell viability and mechanical properties of the various GelMA hydrogels with different concentrations of MA are shown in Figure 6. As displayed in Figure 6A, as the concentration of GelMA remained unchanged, the cell viability of the GelMA hydrogel decreased with an increase in MA concentration. Thus, these results indicated that excessive MA content is not conducive to cell proliferation. This is because an excessive crosslinking density (MA at $8 \%$ ) may form a more dense structure which might restrict cell penetration. ${ }^{41}$ However, cell viability for the 3\% GelMA and 5\% GelMA with different concentrations of MA was not statistically significant in comparison with the $10 \%$ GelMA. Taking all these results into account, the $5 \%$ concentration of GelMA was chosen as the best. Also, we analyzed the optimal MA concentration for cell penetration supporting such results.

To further investigate the inner structure of the 5\% GelMA with different concentrations of MA, SEM images were used to observe the crosslinking effect of the different MA levels on the structure of the GelMA hydrogel (Figure 6B). As shown in Figure 6, a three-dimensional porous structure was observed for the 5\% GelMA with MA-4\% hydrogel. However, the lower concentrations of MA showed a poor crosslinking effect. The results indicated that the 5\% GelMA with MA- $4 \%$ hydrogel had 
A
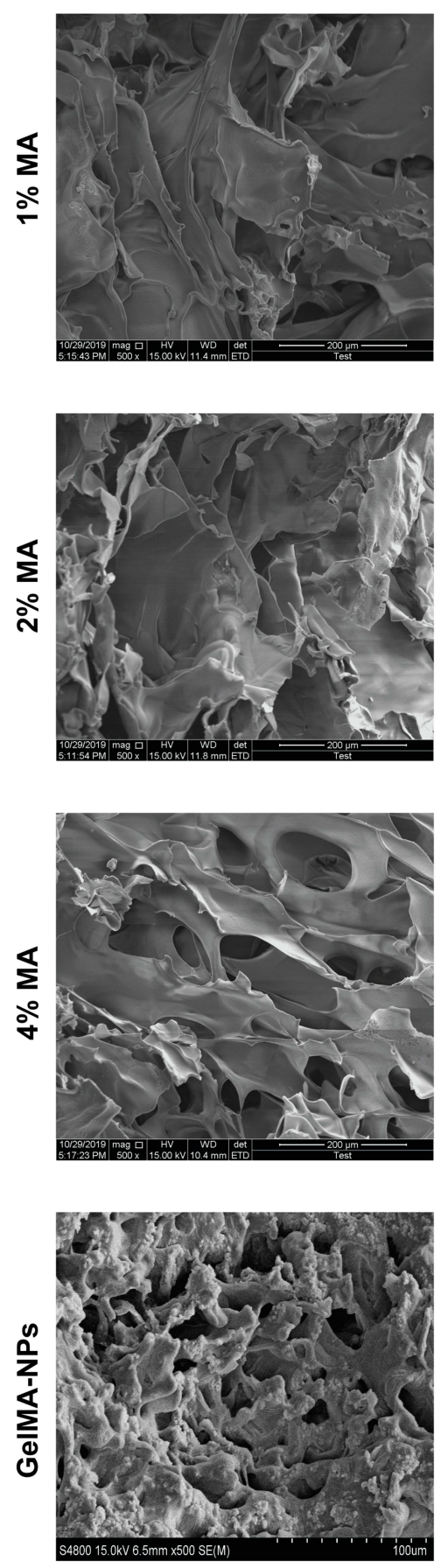

B

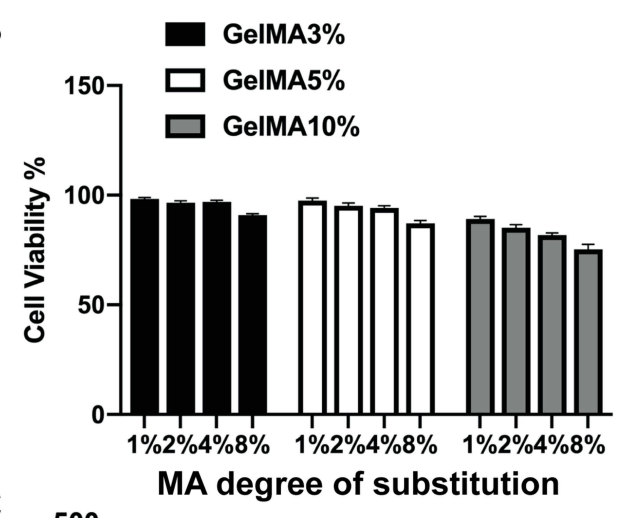

C
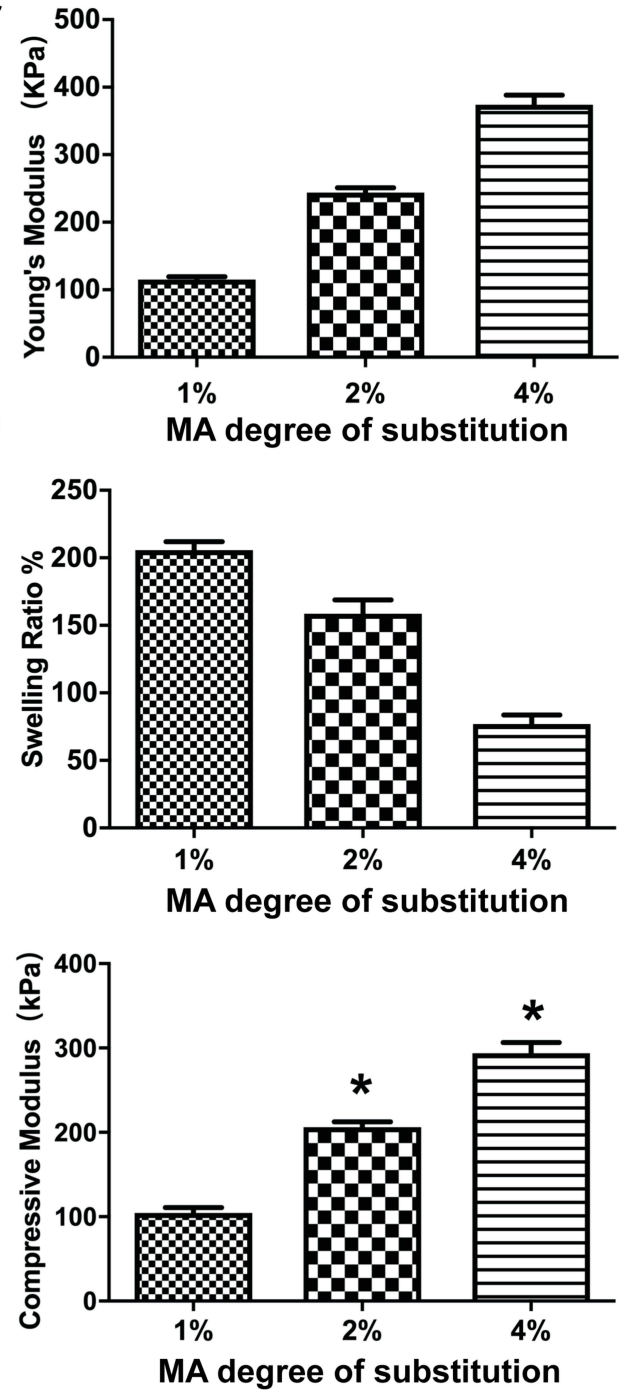

Figure 6 SEM of 5\% GelMA with different concentrations of MA and 5\% GelMA with nanoparticles and 4\% MA (A). The cell viability of the various GelMA with different concentrations of MA (B). The mechanical properties of the $5 \%$ GelMA hydrogel with MA-I\%, MA-2\% and MA-4\% (C and D) and the swelling ratio of the GelMA hydrogels, $5 \%$ GelMA hydrogel with MA-I\%, MA-2\% and MA-4\% (E). ${ }^{*} \mathrm{p}<0.01$ compared to the lowest degree of substitution.

a better crosslinking density and $3 \mathrm{D}$ structure for drug loading and drug delivery.

The mechanical properties of the GelMA hydrogel with various MA content are shown in Figure 6C and D. The Young's modulus and compressive modulus increased with an increase in MA levels. Comparable to the GelMA hydrogel with MA- $1 \%$, the GelMA hydrogel with MA-4\% exhibited a higher Young's modulus and compressive modulus. This finding may be mainly due to the higher degree of substitution of the GelMA hydrogels which resulted in 
A

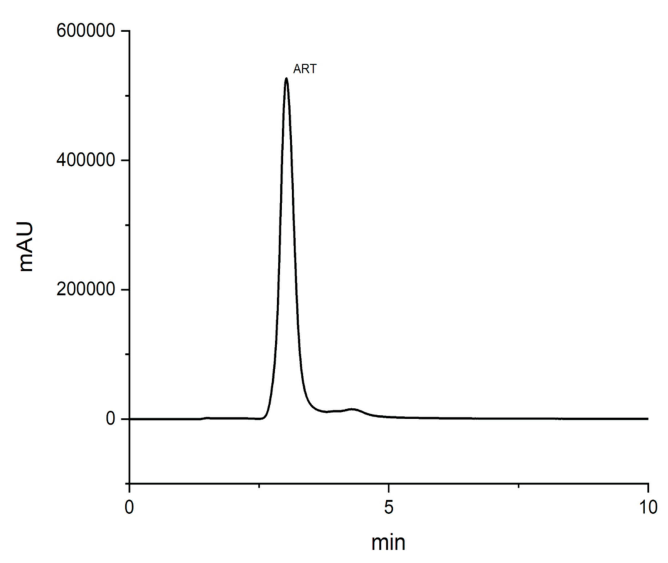

B

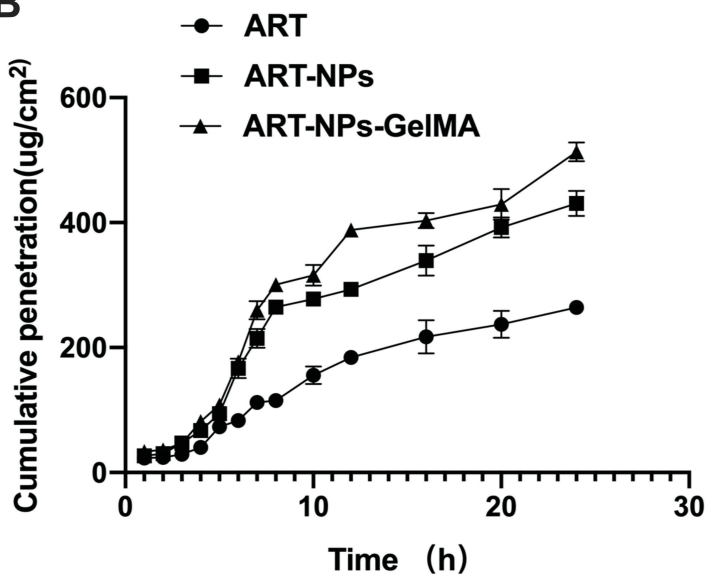

C

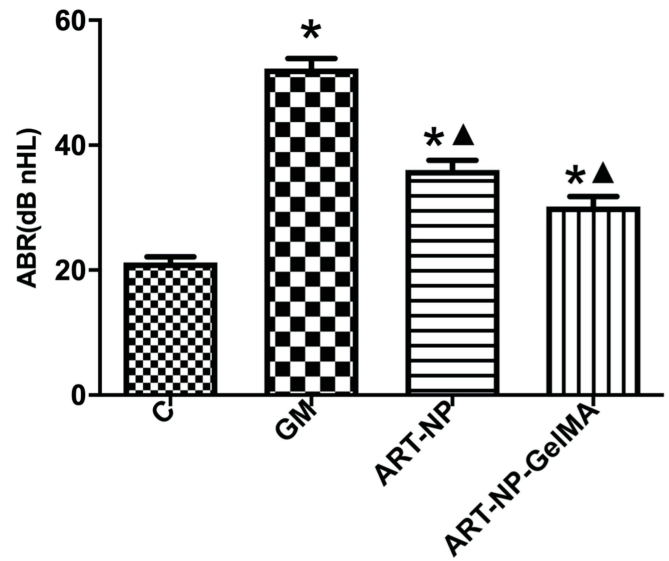

D
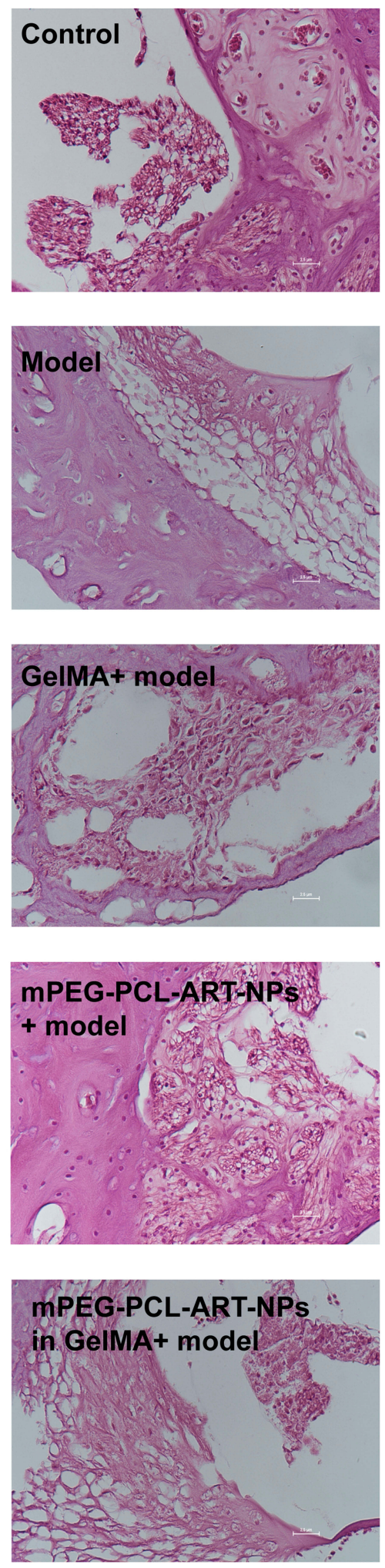

Figure 7 The skin penetration and histological evaluation of the ART-NP-GelMA hydrogel. Chromatogram of ART in receiving the liquid sample (A) and the cumulative penetration of ART, mPEG-PCL-ART-NPs and mPEG-PCL-ART-NPs-GelMA hydrogel at various times (B). Comparison of ABR (C) and representative H\&E stained images of guinea pig cochlea between the control group, GM, GM+ mPEG-ART-NPs, and GM+ ART-NPs-GelMA hydrogel at I4 days later (D). *p<0.0I, compared with control group; $\Lambda_{\mathrm{p}}<0.01$, compared with GM group. Scale bars $=2.5 \mu \mathrm{m}$. 
a much better crosslinking structure. Thus, a higher MA content could lead to enhanced mechanical properties. Therefore, we postulated that the MA concentration of $4 \%$ could be adopted to obtain a stable structure of GelMA for improved drug delivery.

The swelling behavior of the GelMA hydrogels with various MA content is presented in Figure 6E. Indeed, the swelling behavior of the GelMA hydrogels exhibited a trend dependent on the concentration of MA, with swelling decreasing with an increase in the degree of substitution of the GelMA hydrogels. These findings further demonstrated that the degree of substitution of GelMA can significantly influence gelation ability, as well as the propensity for adsorbing water within the polymer network. Usually, the swelling characteristics of a network are important for cell culture as it affects the solute diffusion and surface properties. The degree of substitution of GelMA is correlated to the crosslink density, while a higher degree of substitution of GelMA leads to the formation of hydrogels with a denser network. Thus, an increasing amount of methacrylate groups in the feed composition was expected to enhance the density of the network and subsequently decrease the degree of swelling.

\section{Skin Penetration Capability}

The HPLC method was used to determine the content of ART in the liquid. ART could be detected at $3 \mathrm{~min}$, which may be helpful for rapid detection (Figure 7A). Figure 7B presents the cumulative penetration of ART, the optimal mPEG-PCL-ART-NPs and mPEG-PCL-ART-NPs-GelMA hydrogel at various time periods. It could be observed that the mPEG-PCL-ART-NPs-GelMA hydrogel had a higher cumulative penetration than the ART and mPEG-PCL-ART -NPs. Meanwhile, the PEG-PCL nanoparticles promoted ART skin penetration capability. These results suggest that the PEG-PCL nanoparticle may increase skin adhesive capacity and avoid fast diffusion in the skin.

\section{Pharmacodynamic Study}

As mentioned, a hearing loss model for in vivo ototoxicity was established by applying gentamicin to guinea pigs. As expected, gentamicin induced severe damage to the conductive hearing capacity (Figure 7C). More importantly, the basement membrane morphology was normal and the hair cells were arranged neatly with a normal morphology in the control group (Figure 7D). Besides, there were no exuding red blood cells and the spiral primary band structure was dense which was adjacent to the outer wall of the cochlea. However, in the model and GelMA group, the morphology of the cochlea tissue was obviously changed and damaged, including the fracture of vessels that were thinned and partial dissolved, the outer wall of the cochlea was thickened, and the nuclei of helical nerve cells shrunk and vacuolar degenerated, while the nuclei-plasma ratio was significantly reduced. In the ARTNPs and ART-NPs in GelMA groups, the damage to the inner ear structures were restored with a normal basement membrane structure, normal vascular morphology, only occasional fractures, and basically a normal structure of the helical primary zone, which was continuous with the outer wall of the cochlea without obvious gaps.

Therefore, the present ART-loaded PEG-PCL nanoparticle-based photosensitive hydrogel may be applied for the treatment of hearing loss induced by gentamicin, and, should definitely be further investigated for such applications.

\section{Conclusion}

In the present study, we successfully fabricated a GelMA hydrogel which demonstrated an ability to serve as a drug carrier for mPEG-PCL-ART-NP. The mPEG-PCL nanoparticles showed improved physicochemical properties with enhanced in vitro delivery of ART. Both ART and mPEG-PCL-ART-NPs exhibited better biological activity. Skin penetration and histological evaluation of the mPEGPCL-ART-NPs-GelMA hydrogel showed that ART could effectively diffuse through the skin but generated less damage to the shape of hair cells of the Corti organ. The developed nanodrug hydrogel delivery system should be applied as a potential candidate drug for human clinical trials to reverse hearing loss for patients who receive gentamicin treatments in the not-too distant future.

\section{Funding}

This research was supported by the National Natural Science Foundation of China (No. U1804181), Henan Scientific and Technological Project (No. 182102311187), and the Henan Medical Scientific and Technological Project (No. 2018020396).

\section{Disclosure}

The authors declare no conflicts of interest in this work.

\section{References}

1. Loss H, Aschenbach JR, Ebner F, et al. Effects of a pathogenic ETEC strain and a probiotic Enterococcus faecium for strain on the inflammasome response in porcine dendritic cells. Vet Immunol Immunopathol. 2018;203:78-87. doi:10.1016/j.vetimm.2018.08.004 
2. Yano T, Nishio S, Usami S, et al. Frequency of mitochondrial mutations in non-syndromic hearing loss as well as possibly responsible variants found by whole mitochondrial genome screening. J Hum Genet. 2014;59(2):100-106. doi:10.1038/jhg.2013.128

3. Usami S, Nishio S, Nagano M, et al. Simultaneous screening of multiple mutations by invader assay improves molecular diagnosis of hereditary hearing loss: a multicenter study. PLoS One. 2012;7(2): e31276. doi:10.1371/journal.pone.0031276

4. Miyagawa M, Nishio S, Usami S, et al. Mutation spectrum and genotype-phenotype correlation of hearing loss patients caused by SLC26A4 mutations in the Japanese: a large cohort study. J Hum Genet. 2014;59(5):262-268. doi:10.1038/jhg.2014.12

5. Ernst A, Todt I, Wagner J. Safety and effectiveness of the vibrant soundbridge in treating conductive and mixed hearing loss: a systematic review. Laryngoscope. 2016;126(6):1451-1457. doi:10. 1002/lary. 25670

6. Daniel E. Noise and hearing loss: a review. J Sch Health. 2007;77 (5):225-231. doi:10.1111/j.1746-1561.2007.00197.x

7. Lasak JM, Sataloff RT, Hawkshaw M, et al. Autoimmune inner ear disease: steroid and cytotoxic drug therapy. Ear Nose Throat J. 2001;80(11):808-6, 818. doi:10.1177/014556130108001110

8. Gu L, Cui X, Wei W, et al. Ferulic acid promotes survival and differentiation of neural stem cells to prevent gentamicin-induced neuronal hearing loss. Exp Cell Res. 2017;360(2):257-263. doi:10.1016/j.yexcr.2017.09.015

9. Ma M, Liu X, Tan L, et al. Enhancing the antibacterial efficacy of low-dose gentamicin with 5 minutes assistance of photothermy at 50 oC. Biomater Sci. 2019;7(4):1437-1447. doi:10.1039/C8BM01539B

10. Katva S, Das S, Moti HS, et al. Antibacterial synergy of silver nanoparticles with gentamicin and chloramphenicol against Enterococcus faecalis. Pharmacogn Mag. 2018;13(Suppl 4):S828S833. doi:10.4103/pm.pm_120_17

11. Ström G, Boqvist S, Albihn A, et al. Antimicrobials in small-scale urban pig farming in a lower middle-income country - arbitrary use and high resistance levels. Antimicrob Resist Infect Control. 2018;7 (1):35. doi:10.1186/s13756-018-0328-y

12. Priuska EM, Schacht J. Formation of free radicals by gentamicin and iron and evidence for an iron/gentamicin complex. Biochem Pharmacol. 1995;50(11):1749-1752. doi:10.1016/0006-2952(95)02160-4

13. Sha SH, Schacht J. Salicylate attenuates gentamicin-induced ototoxicity. Lab Invest. 1999;79(7):807-813.

14. Sha S-H, Schacht J. Antioxidants attenuate gentamicin-induced free radical formation in vitro and ototoxicity in vivo: D-methionine is a potential protectant. Hear Res. 2000;142(1):34-40. doi:10.1016/ S0378-5955(00)00003-4

15. Liu L, Liu Y, Ma L, et al. Artemisinin-loaded mesoporous nanoplatform for $\mathrm{pH}$-responsive radical generation synergistic tumor theranostics. ACS Appl Mater Interfaces. 2018;10(7):6155-6167.

16. Elfahmi SS, Chahyadi A. Optimization of genetic transformation of artemisia annua L. using agrobacterium for artemisinin production. Pharmacogn Mag. 2014;10(37):176. doi:10.4103/0973-1296.127372

17. Giannis A, Krieger J, Smeilus T, et al. The antimalarial activity of artemisinin is not stereospecific. Angew Chem. 2018;57 (41):13433-13438. doi:10.1002/anie.201805534

18. Kim JT, Park J-Y, Seo H-S, et al. In vitro antiprotozoal effects of artemisinin on Neospora caninum. Vet Parasitol. 2002;103(1-2):53-63. doi:10.1016/S0304-4017(01)00580-5

19. Efferth T, Romero MR, Wolf DG, et al. The antiviral activities of artemisinin and artesunate. Clin Infect Dis. 2008;47(6):804-811. doi:10.1086/591195

20. Posner GH, McRiner AJ, Paik I-H, et al. Anticancer and antimalarial efficacy and safety of artemisinin-derived trioxane dimers in rodents. J Med Chem. 2004;47(5):1299-1301. doi:10.1021/jm0303711

21. Aderibigbe B. Design of drug delivery systems containing artemisinin and its derivatives. Molecules. 2017;22(2):323. doi:10.3390/ molecules 22020323
22. Chen $\mathrm{Y}$, Lin $\mathrm{X}$, Park H, et al. Study of artemisinin nanocapsules as anticancer drug delivery systems. Nanomedicine. 2009;5(3):316-322. doi:10.1016/j.nano.2008.12.005

23. Manjili HRK, Malvandi H, Mosavi MS, et al. Preparation and physicochemical characterization of biodegradable mPEG-PCL core-shell micelles for delivery of artemisinin. Pharm Sci. 2016;22 (4):234-243. doi:10.15171/PS.2016.37

24. Letchmanan K, Shen S-C, Ng WK, et al. Enhanced dissolution and stability of artemisinin by nano-confinement in ordered mesoporous SBA-15 particles. J Microencapsul. 2015;32(4):390-400.

25. Manjili HK, Malvandi $\mathrm{H}$, Mousavi MS, et al. In vitro and in vivo delivery of artemisinin loaded PCL-PEG-PCL micelles and its pharmacokinetic study. Artif Cells Nanomed Biotechnol. 2018;46 (5):926-936. doi:10.1080/21691401.2017.1347880

26. Chen J, Guo Z, Wang H-B, et al. Multifunctional mesoporous nanoparticles as $\mathrm{pH}$-responsive $\mathrm{Fe} 2+$ reservoirs and artemisinin vehicles for synergistic inhibition of tumor growth. Biomaterials. 2014;35 (24):6498-6507. doi:10.1016/j.biomaterials.2014.04.028

27. Endres TK, Beck-Broichsitter M, Samsonova O, et al. Self-assembled biodegradable amphiphilic PEG-PCL-IPEI triblock copolymers at the borderline between micelles and nanoparticles designed for drug and gene delivery. Biomaterials. 2011;32(30):7721-7731. doi:10. 1016/j.biomaterials.2011.06.064

28. Zhang L, Zhuo C, Hai W, et al. Preparation and evaluation of PCL-PEG-PCL polymeric nanoparticles for doxorubicin delivery against breast cancer. RSC Adv. 2016;6(60):54727-54737. doi:10. 1039/C6RA04687H

29. Jiang CP, Huang JR, Hsieh MF. Fabrication of synthesized PCLPEG-PCL tissue engineering scaffolds using an air pressure-aided deposition system. Rapid Prototyp J. 2011;17(4):288-297. doi:10.1108/13552541111138414

30. Khalid I, Ahmad M, Usman Minhas M, et al. Cross-linked sodium alginate-g-poly(acrylic acid) structure: a potential hydrogel network for controlled delivery of loxoprofen sodium. Adv Polym Technol. 2018;37(4):985-995. doi:10.1002/adv.21747

31. Motealleh A, Kehr NS. Nanocomposite hydrogels and their applications in tissue engineering. Adv Healthc Mater. 2017;6(1):1600938. doi:10.1002/adhm.201600938

32. Guo Y, Yuan T, Xiao Z, et al. Hydrogels of collagen/chondroitin sulfate/hyaluronan interpenetrating polymer network for cartilage tissue engineering. J Mater Sci Mater Med. 2012;23(9):2267-2279. doi:10.1007/s10856-012-4684-5

33. Zhao X, Lang Q, Yildirimer L, et al. Photocrosslinkable gelatin hydrogel for epidermal tissue engineering. Adv Healthc Mater. 2015;5(1):108-118. doi:10.1002/adhm.201500005

34. Xiao S, Zhao T, Wang J, et al. Gelatin methacrylate (GelMA)-based hydrogels for cell transplantation: an effective strategy for tissue engineering. Stem Cell Rev Rep. 2019;15(5):664-679. doi:10.1007/ s12015-019-09893-4

35. Naghizadeh Z, Karkhaneh A, Khojasteh A. Self-crosslinking effect of chitosan and gelatin on alginate based hydrogels: injectable in situ forming scaffolds. Mater Sci Eng C. 2018;89:256-264. doi:10.1016/j. msec.2018.04.018

36. Heyart B, Weidt A, Wisotzki EI, et al. Micropatterning of reagent-free, high energy crosslinked gelatin hydrogels for bioapplications. $J$ Biomed Mater Res B Appl Biomater. 2017;106(1):320-330. doi:10.1002/jbm.b.33849

37. Shin SR, Zihlmann C, Akbari M, et al. Reduced graphene oxide-GelMA hybrid hydrogels as scaffolds for cardiac tissue engineering. Small. 2016;12(27):3677-3689. doi:10.1002/ smll.201600178

38. Pahoff S, Meinert C, Bas O, et al. Effect of gelatin source and photoinitiator type on chondrocyte redifferentiation in gelatin methacryloyl-based tissue-engineered cartilage constructs. J Mater Chem B. 2019;7(10):1761-1772. doi:10.1039/C8TB02607F 
39. M. FM, Ehsan SS, Roberto PL, et al. Interpenetrating network gelatin methacryloyl (GelMA) and pectin-g-PCL hydrogels with tunable properties for tissue engineering. Biomater Sci. 2018;6(11):2938-2950. doi:10.1039/C8BM00474A

40. Loessner D, Meinert C, Kaemmerer E, et al. Functionalization, preparation and use of cell-laden gelatin methacryloyl-based hydrogels as modular tissue culture platforms. Nat Protoc. 2016;11(4):727-746. doi:10.1038/nprot.2016.037
41. Liu W, Heinrich MA, Zhou Y, et al. Extrusion bioprinting of shear-thinning gelatin methacryloyl bioinks. Adv Healthc Mater. 2017;6(12):1601451. doi:10.1002/adhm.201601451

\section{Publish your work in this journal}

The International Journal of Nanomedicine is an international, peerreviewed journal focusing on the application of nanotechnology in diagnostics, therapeutics, and drug delivery systems throughout the biomedical field. This journal is indexed on PubMed Central, MedLine, CAS, SciSearch ${ }^{\mathbb{R}}$, Current Contents ${ }^{\mathbb{R}} /$ Clinical Medicine, $^{-}$
Journal Citation Reports/Science Edition, EMBase, Scopus and the Elsevier Bibliographic databases. The manuscript management system is completely online and includes a very quick and fair peer-review system, which is all easy to use. Visit http://www.dovepress.com/ testimonials.php to read real quotes from published authors. 